CERN-TH/96-140

SISSA $/ 81 / 96 / \mathrm{EP}$

LPTENS $/ 96 / 32$

NSF-ITP-96-20

hep-th/9606087

\title{
Solving the Decompactification Problem in String Theory
}

\author{
E. KIRITSIS ${ }^{1,2}$, C. KOUNNAS ${ }^{1, *}$, P.M. PETROPOULOS ${ }^{1, *}$ \\ and \\ J. RIZOS ${ }^{1,3, \diamond}$ \\ ${ }^{1}$ Theory Division, CERN \\ 1211 Geneva 23, Switzerland \\ and \\ ${ }^{2}$ Institute for Theoretical Physics, University of California \\ Santa Barbara, CA 93106-4030, USA \\ and \\ ${ }^{3}$ International School for Advanced Studies, SISSA \\ Via Beirut 2-4, 34013 Trieste, Italy
}

\begin{abstract}
We investigate heterotic ground states in four dimensions in which $N=4$ supersymmetry is spontaneously broken to $N=2$. The $N=4$ supersymmetry is restored at a decompactification limit corresponding to $m_{3 / 2} \rightarrow 0$. We calculate the full moduli-dependent threshold corrections and confirm that they are suppressed in the decompactification limit, as expected from the restoration of $N=4$ supersymmetry. This should be contrasted with the behaviour of the standard $N=2$ ground states, where the couplings blow up linearly with the volume of the decompactifying manifold. This mechanism provides a solution to the decompactification problem for the gauge coupling constants. We also discuss how the mechanism can be implemented in ground states with lower supersymmetry.
\end{abstract}

CERN-TH/96-140

SISSA $/ 81 / 96 / E P$

LPTENS $/ 96 / 32$

NSF-ITP-96-20

June 1996

To appear in Phys. Lett. B

* On leave from Centre National de la Recherche Scientifique, France.

$\diamond$ On leave from Division of Theoretical Physics, Physics Department, University of Ioannina, Greece. 
One of the important unsolved problems in string theory is supersymmetry breaking, for which there are two known mechanisms.

(i) Tree-level breaking, which comprises Scherk-Schwarz or internal magnetic type of breaking 1, 2]. Such an approach can be implemented in the context of perturbative string theory, but it suffers from the decompactification problem: generically, the gravitino mass, which sets the scale of supersymmetry breaking, is inversely proportional to an internal radius, and when set to be of the order of a $\mathrm{TeV}$, a tower of states (charged under lowenergy gauge groups) populate the energy range between the supersymmetry-breaking scale and the Planck scale. Then low-energy couplings run fast (almost linearly with the radius) and the theory becomes strongly coupled long before the unification (or Planck) scale. This behaviour is thought to be generic, and although there are some ideas on how to avoid itt [3], no workable model exists so far.

(ii) Non-perturbative breaking via gaugino condensation [4]. This is a non-perturbative breaking of supersymmetry, which up till now had to be discussed at the level of the effective supergravity. The problem there was the creation of a runaway potential for the dilaton field, plus our inability to do a controllable calculation.

In this note we will show that in a class of ground states of string theory, which have the structure of spontaneously broken $N=4$ theories, the behaviour of thresholds as functions of the moduli of the internal manifold is radically different from the known examples. The reason for this is that $N=4$ supersymmetry, at large values of (some) moduli, is restored and thus the thresholds vanish in the limit instead of blowing up. Such a behaviour was already anticipated in [5]. Moreover, by using non-perturbative duality techniques, available today, we might also find a similar behaviour in the context of non-perturbatively broken supersymmetry [6].

We will not present here a complete realistic model of broken supersymmetry. We expect however that in such models, viewed as orbifolds of $N=4$ ground states with respect to groups without fixed points (such orbifolds are also known as stringy Scherk-Schwarz ground states [1]), the internal volume dependence of the thresholds comes from $N=2$ sectors. Thus, here we will study $N=2$ ground states that have the structure of spontaneously broken $N=4$ ground states, compute their threshold corrections as well as their universal terms, and show that they have the behaviour advertised above. A complete analysis, including also $N=1$ and $N=0$ ground states, will appear in [6].

The stringy formula for threshold corrections (for supersymmetric ground states and non-anomalous $U(1)$ 's) including a stringy infra-red regularization is [0]-[16]

$$
\frac{16 \pi^{2}}{g_{i}^{2}(\mu)}=k_{i} \frac{16 \pi^{2}}{g_{\text {string }}^{2}}+b_{i} \log \frac{M_{s}^{2}}{\mu^{2}}+\Delta_{i},
$$

\footnotetext{
${ }^{1}$ The idea consists of constructing models without $N=2$ sectors, so that the threshold corrections are independent of the volume moduli of the internal theory.
} 
where, in the $\overline{D R}$ scheme for the effective field theory, the thresholds read:

$$
\Delta_{i}=\int_{\mathcal{F}} \frac{d^{2} \tau}{\operatorname{Im} \tau}\left(\frac{i}{\pi} \frac{1}{|\eta|^{4}} \sum_{a, b=0,1} \frac{\partial_{\tau} \vartheta\left[\begin{array}{l}
a \\
b
\end{array}\right]}{\eta}\left(\bar{P}_{i}^{2}-\frac{k_{i}}{4 \pi \operatorname{Im} \tau}\right) C\left[\begin{array}{l}
a \\
b
\end{array}\right]-b_{i}\right)+b_{i} \log \frac{2 e^{1-\gamma}}{\pi \sqrt{27}}
$$

with

$$
b_{i}=\lim _{\operatorname{Im} \tau \rightarrow \infty} \frac{i}{\pi} \frac{1}{|\eta|^{4}} \sum_{a, b=0,1} \frac{\partial_{\tau} \vartheta\left[\begin{array}{l}
a \\
b
\end{array}\right]}{\eta}\left(\bar{P}_{i}^{2}-\frac{k_{i}}{4 \pi \operatorname{Im} \tau}\right) C\left[\begin{array}{l}
a \\
b
\end{array}\right] .
$$

Here $k_{i}$ is the level of the $i$ th gauge group factor, $b_{i}$ are the full beta-function coefficients, $M_{s}=1 / \sqrt{\alpha^{\prime}}$ is the string scale, $\mu$ is the infra-red scale, $\bar{P}_{i}$ is the charge operator of the gauge group $G_{i}$, and $C\left[\begin{array}{l}a \\ b\end{array}\right]$ is the internal six-dimensional partition function. For conventions see [10, 13].

To start with, we will consider models that come from toroidal compactification of generic six-dimensional $N=1$ string theories. In such cases, there is a universal two-torus, which provides the (perturbative) central charges of the $N=2$ algebra. Therefore (2) becomes

$$
\Delta_{i}=\int_{\mathcal{F}} \frac{d^{2} \tau}{\operatorname{Im} \tau}\left(\frac{\Gamma_{2,2}(T, U)}{\bar{\eta}^{24}}\left(\bar{P}_{i}^{2}-\frac{k_{i}}{4 \pi \operatorname{Im} \tau}\right) \bar{\Omega}-b_{i}\right)+b_{i} \log \frac{2 e^{1-\gamma}}{\pi \sqrt{27}},
$$

where $T$ and $U$ are the complex moduli of the two-torus, $\bar{\Omega}$ is an antiholomorphic function and

$$
\begin{aligned}
\Gamma_{2,2}(T, U)=\sum_{\mathbf{m}, \mathbf{n} \in Z} \exp ( & -2 \pi i \tau\left(m_{1} n_{1}+m_{2} n_{2}\right) \\
& \left.-\frac{\pi \operatorname{Im} \tau}{\operatorname{Im} T \operatorname{Im} U}\left|T n_{1}+T U n_{2}+U m_{1}-m_{2}\right|^{2}\right) .
\end{aligned}
$$

By advocating modular invariance, analytic properties and infra-red finiteness, it is possible to isolate the universal part of the thresholds as follows?:

$$
\Delta_{i}=b_{i} \Delta-k_{i} Y
$$

where

$$
\Delta=-\log \left(4 \pi^{2}|\eta(T)|^{4}|\eta(U)|^{4} \operatorname{Im} T \operatorname{Im} U\right)
$$

and

$$
Y=-\frac{\xi}{12} \int_{\mathcal{F}} \frac{d^{2} \tau}{\operatorname{Im} \tau} \Gamma_{2,2}(T, U)\left(\left(\bar{E}_{2}-\frac{3}{\pi \operatorname{Im} \tau}\right) \frac{\bar{E}_{4} \bar{E}_{6}}{\bar{\eta}^{24}}-\bar{j}+1008\right) .
$$

Here $E_{2 n}$ are the Eisenstein series:

$$
E_{2}=\frac{12}{i \pi} \partial_{\tau} \log \eta=1-24 \sum_{n=1}^{\infty} \frac{n q^{n}}{1-q^{n}}
$$

\footnotetext{
${ }^{2}$ Details can be found in 14,15 .
} 


$$
\begin{gathered}
E_{4}=\frac{1}{2}\left(\vartheta_{2}^{8}+\vartheta_{3}^{8}+\vartheta_{4}^{8}\right)=1+240 \sum_{n=1}^{\infty} \frac{n^{3} q^{n}}{1-q^{n}} \\
E_{6}=\frac{1}{2}\left(\vartheta_{2}^{4}+\vartheta_{3}^{4}\right)\left(\vartheta_{3}^{4}+\vartheta_{4}^{4}\right)\left(\vartheta_{4}^{4}-\vartheta_{2}^{4}\right)=1-504 \sum_{n=1}^{\infty} \frac{n^{5} q^{n}}{1-q^{n}}, \ldots
\end{gathered}
$$

and $\xi$ is a constant that can be expressed in terms of the number of massless vector multiplets $N_{V}$ and hypermultiplets $N_{H}$, by using the relation between gauge and $R^{2}$-term renormalizations [14, 15]. It reads:

$$
\xi=-\frac{1}{264}\left(22-N_{V}+N_{H}\right) .
$$

It is remarkable that the latter is fully determined as a consequence of the anomaly cancellation (gauge, gravitational and mixed) in the underlying six-dimensional theory [17]. Indeed, as long as the number of tensor multiplets is $N_{T}=1$, anomaly cancellation implies that

$$
N_{H}-N_{V}=242
$$

and therefore

$$
\xi=-1 .
$$

Hence, we observe that for all $N=2$ ground states that come from toroidal compactification of a $N=1$ six-dimensional theory, the threshold corrections can be completely determined (eqs. (6), (7), (8) and (144) and do not depend on the details of the six-dimensional theory (moduli included). As an example, consider the case of the $Z_{2}$ orbifold, where we have a gauge group $E_{8} \times E_{7} \times S U(2) \times U(1)^{2}$ and thus $N_{V}=386$. The number of massless hypermultiplets is $N_{H}=628$. Using these numbers in (12) we indeed obtain $\xi=-1$. As expected from supersymmetry, the corresponding universal threshold is twice as big as a single-plane contribution of the symmetric $Z_{2} \times Z_{2}$ orbifold analysed in [13]. These thresholds were further analysed in refs. [14] and [15]. One important feature is that $Y(T, U)$ given in eq. (8) is finite and continuous inside the moduli space, even along enhanced-symmetry planes as $T=U$.

We will now construct different $N=2$ models in four dimensions, which can be represented as ground states where $N=4$ supersymmetry is spontaneously broken to $N=2$. This can be achieved by doing a $Z_{2}$ rotation on the $T^{4}$ accompanied by a $Z_{2}$ translation on the $T^{2}$. There are three choices for the $Z_{2}$ translation on the $T^{2}$ :

(i) $\left|m_{1}, m_{2}, n_{1}, n_{2}\right\rangle \rightarrow(-1)^{m_{1}}\left|m_{1}, m_{2}, n_{1}, n_{2}\right\rangle$,

(ii) $\left|m_{1}, m_{2}, n_{1}, n_{2}\right\rangle \rightarrow(-1)^{m_{2}}\left|m_{1}, m_{2}, n_{1}, n_{2}\right\rangle$,

(iii) $\left|m_{1}, m_{2}, n_{1}, n_{2}\right\rangle \rightarrow(-1)^{m_{1}+m_{2}}\left|m_{1}, m_{2}, n_{1}, n_{2}\right\rangle$;

the corresponding models will be refered to as models I, II and III, respectively. Here the orbifold group acts without fixed points. Consequently the spectrum is in one-to-one correspondence with that of the $N=4 T^{6}$ compactification. This is a spontaneous breaking of $N=4$ to $N=2$ and the mass of the two gravitinos can be computed:

(i) $m_{3 / 2}^{2}=\frac{|U|^{2}}{\operatorname{Im} T \operatorname{Im} U}$ and $N=4$ supersymmetry is restored in the limit $\operatorname{Im} T \rightarrow \infty, U \rightarrow 0$ with $\operatorname{Im} T \operatorname{Im} U$ finite, and $m_{3 / 2}^{2} \rightarrow 0$ as it should;

(ii) $m_{3 / 2}^{2}=\frac{1}{\operatorname{Im} T \operatorname{Im} U}$ and $N=4$ supersymmetry is restored in the $\operatorname{limit} \operatorname{Im} T \rightarrow \infty$, 
$\operatorname{Im} U \rightarrow \infty$ with $\operatorname{Im} T / \operatorname{Im} U$ fixed;

(iii) $m_{3 / 2}^{2}=\frac{1}{\operatorname{Im} T} \inf \left(\frac{1}{\operatorname{Im} U}, \frac{|U|^{2}}{\operatorname{Im} U}\right)$ and $N=4$ supersymmetry is restored when $\operatorname{Im} T \rightarrow \infty$ with $U$ kept fixed.

We should note that there also exist models where the lattice translations act on the winding numbers. In any such model the original duality group $O(2,2 ; Z)$ is broken to a subgroup. We will describe these groups below. The broken transformations map any given model to a different one. For example the duality transformation $U \rightarrow-1 / U$ maps model I to model II.

The heterotic partition functions can be written as

$$
\begin{aligned}
Z^{A}= & \frac{1}{\operatorname{Im} \tau|\eta|^{4}} \frac{1}{2} \sum_{a, b=0}^{1}(-1)^{a+b+a b}\left(\frac{\vartheta\left[\begin{array}{l}
a \\
b
\end{array}\right]}{\eta}\right)^{2} \\
& \frac{1}{2} \sum_{h, g=0}^{1} \frac{\vartheta\left[\begin{array}{l}
a+h \\
b+g
\end{array}\right]}{\eta} \frac{\vartheta\left[\begin{array}{l}
a-h \\
b-g
\end{array}\right]}{\eta} Z_{4,4}\left[\begin{array}{l}
h \\
g
\end{array}\right] Z_{2,2}^{A}\left[\begin{array}{c}
h \\
g
\end{array}\right] \\
& \frac{1}{2} \sum_{\bar{a}, \bar{b}=0}^{1} \frac{\bar{\vartheta}\left[\begin{array}{l}
\bar{a}+h \\
\bar{b}+g
\end{array}\right]}{\bar{\eta}} \frac{\bar{\vartheta}\left[\begin{array}{l}
\bar{a}-h \\
\bar{b}-g
\end{array}\right]}{\bar{\eta}}\left(\frac{\bar{\vartheta}\left[\begin{array}{l}
\bar{a} \\
\bar{b}
\end{array}\right]}{\bar{\eta}}\right)^{6} \frac{1}{2} \sum_{\bar{c}, \bar{d}=0}^{1}\left(\frac{\bar{\vartheta}\left[\begin{array}{l}
\bar{c} \\
\bar{d}
\end{array}\right]}{\bar{\eta}}\right)^{8} ;
\end{aligned}
$$

here the index $A$ labels the three possible translations I, II, III, and

$$
\begin{gathered}
Z_{4,4}\left[\begin{array}{l}
0 \\
0
\end{array}\right]=\frac{\Gamma_{4,4}}{|\eta|^{8}}, \quad Z_{4,4}\left[\begin{array}{l}
0 \\
1
\end{array}\right]=16 \frac{|\eta|^{4}}{\left|\vartheta_{2}\right|^{4}}=\frac{\left|\vartheta_{3} \vartheta_{4}\right|^{4}}{|\eta|^{8}}, \\
Z_{4,4}\left[\begin{array}{l}
1 \\
0
\end{array}\right]=16 \frac{|\eta|^{4}}{\left|\vartheta_{4}\right|^{4}}=\frac{\left|\vartheta_{2} \vartheta_{3}\right|^{4}}{|\eta|^{8}}, \quad Z_{4,4}\left[\begin{array}{l}
1 \\
1
\end{array}\right]=16 \frac{|\eta|^{4}}{\left|\vartheta_{3}\right|^{4}}=\frac{\left|\vartheta_{2} \vartheta_{4}\right|^{4}}{|\eta|^{8}} .
\end{gathered}
$$

The shifted partition functions of the two-torus are

$$
Z_{2,2}^{A}\left[\begin{array}{l}
h \\
g
\end{array}\right](T, U)=\frac{\Gamma_{2,2}^{A}\left[\begin{array}{l}
h \\
g
\end{array}\right](T, U)}{|\eta|^{4}}
$$

where $\Gamma_{2,2}^{A}\left[\begin{array}{l}0 \\ 0\end{array}\right] \equiv \Gamma_{2,2}$ is given in (可), and

(i) $\Gamma_{2,2}^{\mathrm{I}}\left[\begin{array}{l}h \\ g\end{array}\right]$ is obtained from $\Gamma_{2,2}$ by inserting $(-1)^{m_{1} g}$ and shifting $n_{1} \rightarrow n_{1}+h / 2$,

(ii) $\Gamma_{2,2}^{\mathrm{II}}\left[\begin{array}{l}h \\ g\end{array}\right]$ is obtained from $\Gamma_{2,2}$ by inserting $(-1)^{m_{2} g}$ and shifting $n_{2} \rightarrow n_{2}+h / 2$,

(iii) $\Gamma_{2,2}^{\mathrm{III}}\left[\begin{array}{l}h \\ g\end{array}\right]$ is obtained from $\Gamma_{2,2}$ by inserting $(-1)^{\left(m_{1}+m_{2}\right) g}$ and shifting $n_{1} \rightarrow n_{1}+h / 2$ and $n_{2} \rightarrow n_{2}+h / 2$. These partition functions can be obtained from

$$
\begin{array}{r}
\Gamma_{2,2}\left[\begin{array}{l}
h_{1}, h_{2} \\
g_{1}, g_{2}
\end{array}\right]=\sum_{\mathbf{m}, \mathbf{n} \in Z}(-1)^{m_{1} g_{1}+m_{2} g_{2}} \exp \left(-2 \pi i \tau\left(m_{1}\left(n_{1}+\frac{h_{1}}{2}\right)+m_{2}\left(n_{2}+\frac{h_{2}}{2}\right)\right)\right. \\
\left.-\frac{\pi \operatorname{Im} \tau}{\operatorname{Im} T \operatorname{Im} U}\left|T\left(n_{1}+\frac{h_{1}}{2}\right)+T U\left(n_{2}+\frac{h_{2}}{2}\right)+U m_{1}-m_{2}\right|^{2}\right),
\end{array}
$$


where $\left[\begin{array}{l}h_{1}, h_{2} \\ g_{1}, g_{2}\end{array}\right]$ takes the following values: $\left[\begin{array}{l}h, 0 \\ g, 0\end{array}\right],\left[\begin{array}{l}0, h \\ 0, g\end{array}\right]$ and $\left[\begin{array}{l}h, h \\ g, g\end{array}\right]$ for models I, II and III, respectively. We also have the periodicity

$$
\Gamma_{2,2}^{A}\left[\begin{array}{l}
h \\
g
\end{array}\right]=\Gamma_{2,2}^{A}\left[\begin{array}{c}
h+2 \\
g
\end{array}\right]=\Gamma_{2,2}^{A}\left[\begin{array}{c}
h \\
g+2
\end{array}\right],
$$

and the modular properties

$$
\begin{gathered}
\tau \rightarrow \tau+1, \quad Z_{2,2}^{A}\left[\begin{array}{l}
h \\
g
\end{array}\right] \rightarrow Z_{2,2}^{A}\left[\begin{array}{c}
h \\
h+g
\end{array}\right] \\
\tau \rightarrow-\frac{1}{\tau}, \quad Z_{2,2}^{A}\left[\begin{array}{l}
h \\
g
\end{array}\right] \rightarrow Z_{2,2}^{A}\left[\begin{array}{l}
g \\
h
\end{array}\right] .
\end{gathered}
$$

Finally, by straightforward computation we obtain:

$$
\begin{gathered}
\frac{1}{2} \sum_{h, g=0}^{1} \Gamma_{2,2}^{\mathrm{I}}\left[\begin{array}{l}
h \\
g
\end{array}\right](T, U)=\Gamma_{2,2}\left(\frac{T}{2}, 2 U\right) \\
\frac{1}{2} \sum_{h, g=0}^{1} \Gamma_{2,2}^{\mathrm{II}}\left[\begin{array}{l}
h \\
g
\end{array}\right](T, U)=\Gamma_{2,2}\left(\frac{T}{2}, \frac{U}{2}\right) \\
\frac{1}{2} \sum_{h, g=0}^{1} \Gamma_{2,2}^{\mathrm{III}}\left[\begin{array}{l}
h \\
g
\end{array}\right](T, U)=\Gamma_{2,2}\left(\frac{T}{2}, \frac{1+U}{1-U}\right),
\end{gathered}
$$

which will be useful further on.

The above models have the same gauge group as that of the standard $Z_{2}$ orbifold limit of $K_{3}$ so that $N_{V}=386$. However the number of massless hypermultiplets is different. Compared with the usual $Z_{2}$ orbifold model, the massless hypermultiplets coming from the twisted sector have become massive here. Thus the massless ones are 4 singlets under $E_{8} \times E_{7} \times S U(2)$ and one which is singlet under $E_{8}$ and transforms under $E_{7} \times S U(2)$ as $(56,2)$; then $N_{H}=116$. This should be compared with the standard $Z_{2}$ orbifold, which contains extra massless hypermultiplets coming from the twisted sector: 8 transforming as $(56,1)$ and 32 as $(1,2)$ under $E_{7} \times S U(2)$.

A comment is also in order here concerning the duality symmetries of these models. The standard $Z_{2}$ orbifold has an $O(2,2 ; Z)$ duality symmetry acting on the $T, U$ moduli, which is generated by the standard $S L(2 ; Z)$ transformations on both $T$ and $U$ plus the $T \leftrightarrow U$ interchange transformation. The duality group in this case can be found from the explicit form of the $O(2,2 ; Z)$ transformations:

$$
S L(2 ; Z)_{T}:\left(\begin{array}{c}
m_{1} \\
m_{2} \\
n_{1} \\
n_{2}
\end{array}\right) \rightarrow\left(\begin{array}{rrrr}
d & 0 & 0 & b \\
0 & d & -b & 0 \\
0 & -c & a & 0 \\
c & 0 & 0 & a
\end{array}\right)\left(\begin{array}{c}
m_{1} \\
m_{2} \\
n_{1} \\
n_{2}
\end{array}\right), \quad a d-b c=1,
$$




$$
S L(2 ; Z)_{U}:\left(\begin{array}{c}
m_{1} \\
m_{2} \\
n_{1} \\
n_{2}
\end{array}\right) \rightarrow\left(\begin{array}{rrrr}
a^{\prime} & -c^{\prime} & 0 & 0 \\
-b^{\prime} & d^{\prime} & 0 & 0 \\
0 & 0 & d^{\prime} & b^{\prime} \\
0 & 0 & c^{\prime} & a^{\prime}
\end{array}\right)\left(\begin{array}{c}
m_{1} \\
m_{2} \\
n_{1} \\
n_{2}
\end{array}\right), \quad a^{\prime} d^{\prime}-b^{\prime} c^{\prime}=1
$$

and

$$
T \leftrightarrow U:\left(\begin{array}{c}
m_{1} \\
m_{2} \\
n_{1} \\
n_{2}
\end{array}\right) \rightarrow\left(\begin{array}{llll}
0 & 0 & 1 & 0 \\
0 & 1 & 0 & 0 \\
1 & 0 & 0 & 0 \\
0 & 0 & 0 & 1
\end{array}\right)\left(\begin{array}{c}
m_{1} \\
m_{2} \\
n_{1} \\
n_{2}
\end{array}\right)
$$

The above transformations can be summarized as follows:

$$
\left(\begin{array}{c}
\mathbf{m} \\
\mathbf{n}
\end{array}\right) \rightarrow\left(\begin{array}{cc}
A & B \\
C & D
\end{array}\right)\left(\begin{array}{c}
\mathbf{m} \\
\mathbf{n}
\end{array}\right), \quad A, B, C, D \in G L(2 ; Z)
$$

with

$$
C^{T} A+A^{T} C=0, \quad D^{T} B+B^{T} D=0, \quad C^{T} B+A^{T} D=1 .
$$

(i) For model I the duality group is $\Gamma(2)_{T}$, defined by $b$ even, times $\Gamma(2)_{U}$, defined by $c^{\prime}$ even. In the notation of (28) this corresponds to $A_{12}, B_{12}, B_{21}, D_{21}$ being even and $B_{11}=0 \bmod (4)$. (ii) For model II the duality group is $\Gamma(2)_{T}$, defined by $b$ even, times $\Gamma(2)_{U}$, defined by $b^{\prime}$ even as well as the $T \leftrightarrow U$ interchange. In the notation of (28) this corresponds to $A_{21}, B_{12}, B_{21}, D_{12}$ being even and $B_{22}=0 \bmod (4)$.

(iii) For model III the duality group is $\Gamma(2)_{T}$ defined by $b$ even, times $\tilde{\Gamma}_{U}$. The group $\tilde{\Gamma}$ is defined by the integer matrices

$$
\left(\begin{array}{cc}
m & 2 n+m+1 \\
2 r-m+1 & 2 s-m
\end{array}\right)
$$

with determinant one. In the notation of (28) this corresponds to $A_{11}+A_{12}$ and $A_{21}+A_{22}$ being simultaneously even or simultaneously odd, $D_{11}+D_{12}$ and $D_{21}+D_{22}$ being simultaneously even or simultaneously odd, and $B_{11}+B_{12}+B_{21}+B_{22}$ vanishing modulo 4 together with the other combinations where two signs are flipped.

We will now evaluate the threshold corrections for the above three models. Using eq. (2) we obtain]:

$$
\Delta_{i}^{A}=\int_{\mathcal{F}} \frac{d^{2} \tau}{\operatorname{Im} \tau}\left(-\sum_{(h, g)}^{\prime} \frac{\Gamma_{2,2}^{A}\left[\begin{array}{l}
h \\
g
\end{array}\right]}{\bar{\eta}^{24}}\left(\bar{P}_{i}^{2}-\frac{k_{i}}{4 \pi \operatorname{Im} \tau}\right) \bar{\Omega}\left[\begin{array}{l}
h \\
g
\end{array}\right]-b_{i}\right)+b_{i} \log \frac{2 e^{1-\gamma}}{\pi \sqrt{27}}
$$

with

$$
\begin{aligned}
\Omega\left[\begin{array}{l}
0 \\
1
\end{array}\right] & =\frac{1}{2} E_{4} \vartheta_{3}^{4} \vartheta_{4}^{4}\left(\vartheta_{3}^{4}+\vartheta_{4}^{4}\right) \\
\Omega\left[\begin{array}{l}
1 \\
0
\end{array}\right] & =-\frac{1}{2} E_{4} \vartheta_{2}^{4} \vartheta_{3}^{4}\left(\vartheta_{2}^{4}+\vartheta_{3}^{4}\right) \\
\Omega\left[\begin{array}{l}
1 \\
1
\end{array}\right] & =-\frac{1}{2} E_{4} \vartheta_{2}^{4} \vartheta_{4}^{4}\left(\vartheta_{2}^{4}-\vartheta_{4}^{4}\right) .
\end{aligned}
$$

\footnotetext{
${ }^{3}$ The prime summation over $(h, g)$ stands for $(h, g)=\{(0,1),(1,0),(1,1)\}$.
} 
These functions obey the following identity:

$$
\sum_{(h, g)}^{\prime} \Omega\left[\begin{array}{l}
h \\
g
\end{array}\right]=E_{4} E_{6}
$$

We proceed by using arguments similar to those that were used in refs. [14, 15] in order to reach (8) starting from (4). The thresholds (31) can be written in the form

$$
\Delta_{i}^{A}=\int_{\mathcal{F}} \frac{d^{2} \tau}{\operatorname{Im} \tau}\left(\bar{F}_{i}^{A}-b_{i}\right)+b_{i} \log \frac{2 e^{1-\gamma}}{\pi \sqrt{27}}
$$

with

$$
\begin{aligned}
\bar{F}_{i}^{A} & =-k_{i} \sum_{(h, g)}^{\prime} \frac{\Gamma_{2,2}^{A}\left[\begin{array}{l}
h \\
g
\end{array}\right]}{\bar{\eta}^{24}}\left(\frac{\bar{E}_{2}}{12}-\frac{1}{4 \pi \operatorname{Im} \tau}\right) \bar{\Omega}\left[\begin{array}{l}
h \\
g
\end{array}\right]-\sum_{(h, g)}^{\prime} \frac{\Gamma_{2,2}^{A}\left[\begin{array}{l}
h \\
g
\end{array}\right]}{\bar{\eta}^{24}}\left(\bar{P}_{i}^{2}-\frac{k_{i} \bar{E}_{2}}{12}\right) \bar{\Omega}\left[\begin{array}{l}
h \\
g
\end{array}\right] \\
& =k_{i} \bar{F}_{\text {grav }}^{A}+\sum_{(h, g)}^{\prime} \Gamma_{2,2}^{A}\left[\begin{array}{l}
h \\
g
\end{array}\right] \bar{\Lambda}_{i}\left[\begin{array}{l}
h \\
g
\end{array}\right] .
\end{aligned}
$$

Here we have separated the universal term

$$
\bar{F}_{\text {grav }}^{A}=-\sum_{(h, g)}^{\prime} \frac{\Gamma_{2,2}^{A}\left[\begin{array}{l}
h \\
g
\end{array}\right]}{\bar{\eta}^{24}}\left(\frac{\bar{E}_{2}}{12}-\frac{1}{4 \pi \operatorname{Im} \tau}\right) \bar{\Omega}\left[\begin{array}{l}
h \\
g
\end{array}\right]
$$

which is the associated function that appears in the $R^{2}$-term renormalization, and

$$
\bar{\Lambda}_{i}\left[\begin{array}{l}
h \\
g
\end{array}\right]=-\frac{1}{\bar{\eta}^{24}}\left(\bar{P}_{i}^{2}-\frac{k_{i} \bar{E}_{2}}{12}\right) \bar{\Omega}\left[\begin{array}{l}
h \\
g
\end{array}\right] .
$$

For the models under consideration, $\Lambda_{i}\left[\begin{array}{l}h \\ g\end{array}\right]$ can be expressed as $\Lambda_{i}\left[\begin{array}{l}0 \\ 1\end{array}\right]=f_{i}(1-x), \Lambda_{i}\left[\begin{array}{l}1 \\ 0\end{array}\right]=f_{i}(x)$, $\Lambda_{i}\left[\begin{array}{l}1 \\ 1\end{array}\right]=f_{i}\left(\frac{x}{x-1}\right)$, where $x=\left(\vartheta_{2} / \vartheta_{3}\right)^{4}$ and

$$
f_{i}(x)=b_{i}-k_{i} \rho(x)+\left(\frac{\tilde{b}_{i}}{3}-b_{i}-40 k_{i}\right) \sigma(x)
$$

with

$$
\begin{aligned}
\rho(x) & =\frac{4}{3 x(x-1)^{2}}\left(8-49 x+66 x^{2}-49 x^{3}+8 x^{4}\right) \\
\sigma(x) & =-\frac{(x-1)^{2}}{x} .
\end{aligned}
$$

The constants $b_{i}$ are the beta-function coefficients of the models $\left(b_{E_{8}}=-60, b_{E_{7}}=-12\right.$, $\left.b_{S U(2)}=52\right)$ while $\tilde{b}_{i}$ are the beta-function coefficients of the symmetric $Z_{2}$ orbifold $\left(\tilde{b}_{E_{8}}=\right.$ $\left.-60, \tilde{b}_{E_{7}}=\tilde{b}_{S U(2)}=84\right)$. Note also the identities ${ }^{4}$

$$
\sum_{(h, g)}^{\prime} \sigma\left[\begin{array}{l}
h \\
g
\end{array}\right]=3
$$

\footnotetext{
${ }^{4}$ We use the notation $\sigma\left[\begin{array}{l}0 \\ 1\end{array}\right]=\sigma(1-x), \sigma\left[\begin{array}{l}1 \\ 0\end{array}\right]=\sigma(x), \sigma\left[\begin{array}{l}1 \\ 1\end{array}\right]=\sigma\left(\frac{x}{x-1}\right)$, and similarly for $\rho(x)$.
} 


$$
\sum_{(h, g)}^{\prime} \rho\left[\begin{array}{l}
h \\
g
\end{array}\right]=-\frac{j}{12}-36
$$

which imply

$$
\sum_{(h, g)}^{\prime} f_{i}\left[\begin{array}{l}
h \\
g
\end{array}\right]=k_{i}\left(\frac{j}{12}-84\right)+\tilde{b}_{i}
$$

and lead to the correct result (7), (8) for the symmetric $Z_{2}$ orbifold obtained when the substitution $\Gamma_{2,2}^{A}\left[\begin{array}{l}h \\ g\end{array}\right] \rightarrow \Gamma_{2,2}$ is performed.

Using the above results, the thresholds (36) take the form

$$
\Delta_{i}^{A}=b_{i} \Delta^{A}+\left(\frac{\tilde{b}_{i}}{3}-b_{i}\right) \delta^{A}-k_{i} Y^{A}
$$

where

$$
\begin{aligned}
\Delta^{A} & =\int_{\mathcal{F}} \frac{d^{2} \tau}{\operatorname{Im} \tau}\left(\sum_{(h, g)}^{\prime} \Gamma_{2,2}^{A}\left[\begin{array}{l}
h \\
g
\end{array}\right]-1\right)+\log \frac{2 e^{1-\gamma}}{\pi \sqrt{27}} \\
\delta^{A} & =\int_{\mathcal{F}} \frac{d^{2} \tau}{\operatorname{Im} \tau} \sum_{(h, g)}^{\prime} \Gamma_{2,2}^{A}\left[\begin{array}{l}
h \\
g
\end{array}\right] \bar{\sigma}\left[\begin{array}{l}
h \\
g
\end{array}\right] \\
Y^{A} & =\int_{\mathcal{F}} \frac{d^{2} \tau}{\operatorname{Im} \tau} \sum_{(h, g)}^{\prime} \Gamma_{2,2}^{A}\left[\begin{array}{l}
h \\
g
\end{array}\right]\left(\frac{1}{\bar{\eta}^{24}}\left(\frac{E_{2}}{12}-\frac{1}{4 \pi \operatorname{Im} \tau}\right) \bar{\Omega}\left[\begin{array}{l}
h \\
g
\end{array}\right]+\bar{\rho}\left[\begin{array}{l}
h \\
g
\end{array}\right]+40 \bar{\sigma}\left[\begin{array}{l}
h \\
g
\end{array}\right]\right) .
\end{aligned}
$$

Before any further computation we should address two issues here. First we observe that the threshold corrections in these models cannot be decomposed in the simple form $b_{i} \Delta+$ $k_{i} Y$, as was described earlier for $N=2$ models coming from toroidal compactification of sixdimensional theories. The other issue is why the beta-function coefficients $\tilde{b}_{i}$ of the previous models come in the thresholds here. This is not difficult to understand, once we consider some decompactification limits of the present models. The first is the supersymmetry restoration limit $m_{3 / 2} \rightarrow 0$. In this case we obtain a $N=4$ theory in five or six dimensions. There is, however, another decompactification limit, namely $m_{3 / 2} \rightarrow \infty$. There we obtain a sixdimensional $N=1$ theory, which is exactly the same as the one relevant for the original models. The above statements come from the following decompactification limits of the two-torus blocks. Consider (18) in the Lagrangian representation:

$$
\operatorname{Im} \tau \Gamma_{2,2}\left[\begin{array}{c}
h_{1}, h_{2} \\
g_{1}, g_{2}
\end{array}\right]=\sqrt{\operatorname{det} G} \sum_{\mathbf{m}, \mathbf{n} \in Z} e^{-\frac{\pi}{\operatorname{Im} \tau} \sum_{i, j}\left(G_{i j}+B_{i j}\right)\left[m_{i}+\frac{g_{i}}{2}+\left(n_{i}+\frac{h_{i}}{2}\right) \tau\right]\left[m_{j}+\frac{g_{j}}{2}+\left(n_{j}+\frac{h_{j}}{2}\right) \bar{\tau}\right]}
$$

where as usual

$$
G=\frac{\operatorname{Im} T}{\operatorname{Im} U}\left(\begin{array}{cc}
1 & \operatorname{Re} U \\
\operatorname{Re} U & |U|^{2}
\end{array}\right), \quad B=\operatorname{Re} T\left(\begin{array}{rr}
0 & -1 \\
1 & 0
\end{array}\right)
$$

It is easy to verify that as $\operatorname{Im} T \rightarrow \infty$ :

$$
\operatorname{Im} \tau \Gamma_{2,2}\left[\begin{array}{l}
h_{1}, h_{2} \\
g_{1}, g_{2}
\end{array}\right] \rightarrow\left\{\begin{array}{l}
\operatorname{Im} T \text { for } h_{i}, g_{i}=0, \\
\text { exponentially suppressed otherwise . }
\end{array}\right.
$$


Using the dual partition function we can check that in the opposite $\operatorname{limit}, \operatorname{Im} T \rightarrow 0$ :

$$
\operatorname{Im} \tau \Gamma_{2,2}\left[\begin{array}{c}
h_{1}, h_{2} \\
g_{1}, g_{2}
\end{array}\right] \rightarrow \frac{1}{\operatorname{Im} T} \forall h_{i}, g_{i}
$$

However, from the six-dimensional view point, the beta-function coefficients $\tilde{b}_{i}$ are related to anomaly coefficients in that theory (see for example [18]). Consider the anomaly eight-form in six dimensions $I_{8}=X_{4} \tilde{X}_{4}$ with

$$
X_{4}=\frac{1}{4(2 \pi)^{2}}\left(\operatorname{tr} R^{2}-\sum_{i} v_{i} \operatorname{tr} F_{i}^{2}\right), \quad \tilde{X}_{4}=\frac{1}{4(2 \pi)^{2}}\left(\operatorname{tr} R^{2}-\sum_{i} \tilde{v}_{i} \operatorname{tr} F_{i}^{2}\right) .
$$

The first term appears in the six-dimensional Bianchi identity, $d H=\alpha^{\prime}(2 \pi)^{2} X_{4}$ while the second is the one-loop correction of the field equation $d \star H=\alpha^{\prime}(2 \pi)^{2} \tilde{X}_{4}$. The coefficients $v_{i}$ are related to the tree-level value of the gauge couplings, namely $v_{i}=k_{i} / c_{i}$ with $c_{i}=$ $2,1, \frac{1}{3}, \frac{1}{6}, \frac{1}{30}$ for $S U(N), S O(2 N), E_{6,7,8}$. The coefficients $\tilde{v}_{i}$ are determined from GreenSchwarz anomaly cancellation, and the corresponding four-dimensional $N=2$ beta-function coefficients are given by

$$
\tilde{b}_{i}=12\left(1+\frac{\tilde{v}_{i}}{v_{i}}\right)
$$

This explains their appearance in our models.

The integrals in (45) can be explicitly evaluated using the results of [8, 12], (22)-(24) and the duplication formulae for the $\vartheta$-functions with the result

$$
\begin{aligned}
\Delta^{\mathrm{I}} & =-\log \left(\frac{\pi^{2}}{4}\left|\vartheta_{4}(T)\right|^{4}\left|\vartheta_{2}(U)\right|^{4} \operatorname{Im} T \operatorname{Im} U\right) \\
\Delta^{\mathrm{II}} & =-\log \left(\frac{\pi^{2}}{4}\left|\vartheta_{4}(T)\right|^{4}\left|\vartheta_{4}(U)\right|^{4} \operatorname{Im} T \operatorname{Im} U\right) \\
\Delta^{\mathrm{III}} & =-\log \left(\frac{\pi^{2}}{4}\left|\vartheta_{4}(T)\right|^{4}\left|\vartheta_{3}(U)\right|^{4} \operatorname{Im} T \operatorname{Im} U\right) .
\end{aligned}
$$

These thresholds should be contrasted with (7). For both decompactification $\operatorname{limits} \operatorname{Im} T \rightarrow$ $\infty$ and $\operatorname{Im} U \rightarrow \infty$ in (7), we obtain the familiar result that the thresholds grow linearly with the appropriate modulus. To simplify matters we will take $\operatorname{Re} T=\operatorname{Re} U=0$ so that $\operatorname{Im} T=R_{1} R_{2}, \operatorname{Im} U=R_{2} / R_{1}$, and the two-torus decouples into two circles with radii $R_{1}$ (associated with $m_{1}, n_{1}$ ) and $R_{2}$ (associated with $m_{2}, n_{2}$ ), respectively. The thresholds for the original models take the form $\left(R_{1}=R_{2}=R\right)$

$$
\lim _{R \rightarrow \infty} \Delta=\frac{\pi}{3} R^{2}-\log R^{2}+\mathcal{O}(1) .
$$

\footnotetext{
${ }^{5}$ Similar non-universal thresholds in the context of $N=1$ models have also been computed (and presented in a different form) in [11].
} 
This is the expected geometric behaviour of the running gauge coupling. Similarly we also have, for the universal contribution [13]:

$$
\lim _{R \rightarrow \infty} Y=4 \pi R^{2}+\mathcal{O}\left(\frac{1}{R^{2}}\right)
$$

Notice the absence of the logarithmic piece in (56). We will also consider for the sake of comparison the limit $R_{1} \rightarrow \infty$ for fixed $R_{2}$. We obtain:

$$
\lim _{R_{1} \rightarrow \infty} \Delta=\frac{\pi}{3}\left(R_{2}+\frac{1}{R_{2}}\right) R_{1}-\log R_{1}^{2}+\mathcal{O}(1)
$$

while for the universal term

$$
\lim _{R_{1} \rightarrow \infty} Y=\frac{4 \pi}{3} R_{1}\left(3 R_{2}+\frac{1}{R_{2}^{3}}\right) \Theta\left(R_{2}-1\right)+\frac{4 \pi}{3} R_{1}\left(\frac{3}{R_{2}}+R_{2}^{3}\right) \Theta\left(1-R_{2}\right)+\mathcal{O}\left(\frac{1}{R_{1}^{2}}\right) .
$$

Let us now compute the large-radius limits of the thresholds (45). For model I, the supersymmetry restoration limit is $R_{1} \rightarrow \infty\left(m_{3 / 2}=1 / R_{1}\right)$. In this limit we obtain:

$$
\begin{aligned}
\lim _{R_{1} \rightarrow \infty} \Delta^{\mathrm{I}} & =-\log \left(R_{1}^{2}\right)+\mathcal{O}(1) \\
\lim _{R_{1} \rightarrow \infty} \delta^{\mathrm{I}} & =\mathcal{O}\left(e^{-R_{1}^{2}}\right) .
\end{aligned}
$$

As expected we no longer have the linear explosion of the threshold correction as in (57), but we obtain the (apparently) counter-intuitive result that the threshold correction diverges logarithmically. Indeed, we would expect that since in this limit $N=4$ supersymmetry is restored, the thresholds should vanish. Below we will resolve this discrepancy, which is an infra-red phenomenon. Notice, however, that since the universal contribution is infra-redfinite, it vanishes in the restoration limit, as it should:

$$
\lim _{R_{1} \rightarrow \infty} Y^{\mathrm{I}}=\mathcal{O}\left(\frac{1}{R_{1}^{2}}\right)
$$

The asymptotic expressions are similar for model II (with $R_{1} \leftrightarrow R_{2}$ ) while for model III, where both $R_{1} \rightarrow \infty$ and $R_{2} \rightarrow \infty$, we get

$$
\begin{aligned}
\lim _{R_{1,2} \rightarrow \infty} \Delta^{\mathrm{III}} & =-\log \left(R_{1} R_{2}\right)+\mathcal{O}(1) \\
\lim _{R_{1,2} \rightarrow \infty} \delta^{\mathrm{III}} & =\mathcal{O}\left(e^{-R_{1} R_{2}}\right) \\
\lim _{R_{1,2} \rightarrow \infty} Y^{\mathrm{III}} & =\mathcal{O}\left(\frac{1}{R_{1} R_{2}}\right) .
\end{aligned}
$$

Note also that in the opposite limit $m_{3 / 2} \rightarrow \infty$ the thresholds match those (55)-(58) of the previously described models [14, 15].

Let us now concentrate on the issue of how to take various infra-red limits. Consider turning on all Wilson lines which break the gauge group to $U(1)$ 's. All charged states are 
now massive. Some of these states in general have masses of the same order as $m_{3 / 2}$, or above, and some others have masses below $m_{3 / 2}$. Suppose first that there are no states with masses lower than $m_{3 / 2}$. In this case, as $m_{3 / 2} \rightarrow 0$, all thresholds would vanish in the limit. In the case where there are states that are always lighter than $m_{3 / 2}$ as $m_{3 / 2} \rightarrow 0$, these states have always an effective $N=2$ behaviour and they will produce a logarithmic running even when $m_{3 / 2}=0$. In the case we considered, the charged massless states produce an infra-red divergence proportional to $\log \mu^{2}$, which, when present, implies also the (non-holomorphic) logarithmic factor $\log (\operatorname{Im} T \operatorname{Im} U)$.

The question we should answer is: Can we turn Wilson lines so that all charged states become heavier than $m_{3 / 2}$ ? The answer is yes. Consider for simplicity model I, where the gravitino mass is (in the presence of Wilson lines $W_{i}=Y_{i}^{1}+U Y_{i}^{2}$ ):

$$
m_{3 / 2}^{2}=\frac{|U|^{2}}{\operatorname{Im} T \operatorname{Im} U-\sum_{i} \operatorname{Im} W_{i}^{2}} .
$$

The masses of charged particles, which become massless for zero Wilson lines, are

$$
m_{\text {charged }}^{2}=\frac{\left|W^{i} q_{i}\right|^{2}}{\operatorname{Im} T \operatorname{Im} U-\sum_{i} \operatorname{Im} W_{i}^{2}} .
$$

where $q_{i}$ are integers. Due to duality symmetries, the Wilson lines have the periodicity properties $W_{i} \sim W_{i}+1 \sim W_{i}+U$. It is now obvious that for finite $Y_{i}^{1}$, in the supersymmetry restoration limit $U \rightarrow 0$, all charged particles become heavier than the gravitinos. Similar remarks apply to the other models.

For practical purposes, the supersymmetry-breaking scale is a tiny fraction of the Planck mass and there are few states that lie below it. Thus, even in such a case there is no decompactification problem. There is only the logarithmic running of the light particles up to the supersymmetry-breaking scale. Anything above does not run since the physics above $m_{3 / 2}$ is governed by $N=4$ supersymmetry.

Can this mechanism work in models with lower supersymmetry? In the most general case we could imagine three ordered supersymmetry-breaking scales, $m_{0} \leq m_{1} \leq m_{2}$. At scales above $m_{2}$ we have restored $N=4$ supersymmetry. In the range $m_{1} \leq \mu \leq m_{2}$ supersymmetry is spontaneously broken to $N=2$ while for $m_{0} \leq \mu \leq m_{1}$ it is spontaneously broken to $N=1$. Finally, below $m_{0}$ all supersymmetries are broken. It is possible that some of these scales are the same, e.g. $m_{0}=m_{1}$. If $m_{2}$ is sufficiently low then there is no decompactification problem in the theory. It remains to be seen if realistic models can be constructed with low values of $m_{2}$.

\section{Acknowledgements}

E. Kiritsis was supported in part by the grant PHY94-07194. He would also like to thank the ITP for the warm hospitality extended to him during his stay there, while part of this work was done. C. Kounnas was supported in part by EEC contracts SC1*-0394C and SC1*-CT92-0789. J. Rizos would like to thank the CERN Theory Division for hospitality and acknowledges financial support from the EEC contract ERBCHBGCT940634. 


\section{References}

[1] C. Kounnas and M. Porrati, Nucl. Phys. B310 (1988) 355;

S. Ferrara, C. Kounnas, M. Porrati and F. Zwirner, Nucl. Phys. B318 (1989) 75;

I. Antoniadis, C. Bachas, D. Lewellen and T. Tomaras, Phys. Lett. 207B (1988) 441;

C. Kounnas and B. Rostand, Nucl. Phys. B341 (1990) 641;

I. Antoniadis, Phys. Lett. 246B (1990) 377;

I. Antoniadis and C. Kounnas, Phys. Lett. 261B (1991) 369.

[2] C. Bachas, hep-th/9503030.

[3] I. Antoniadis, Phys. Lett. 246B (1990) 377;

K. Benakli, hep-th/9509115.

[4] H.-P. Nilles, Phys. Lett. 115B (1982) 193 and Nucl. Phys. B217 (1983) 366;

S. Ferrara, L. Girardello and H.-P. Nilles, Phys. Lett. 125B (1983) 457;

J.-P. Derendinger, L.E. Ibáñez and H.-P. Nilles, Phys. Lett. 155B (1985) 65;

M. Dine, R. Rohm, N. Seiberg and E. Witten, Phys. Lett. 156B (1985) 55;

C. Kounnas and M. Porrati, Phys. Lett. 191B (1987) 91.

[5] S. Ferrara, C. Kounnas and F. Zwirner, Nucl. Phys. B429 (1994) 589 and erratum, ibid. B433 (1995) 255.

[6] E. Kiritsis and C. Kounnas, CERN-TH/96-134;

E. Kiritsis, C. Kounnas, P.M. Petropoulos and J. Rizos, in preparation.

[7] V. Kaplunovsky, Nucl. Phys. B307 (1988) 145 and erratum, ibid. B382 (1992) 436.

[8] L. Dixon, V. Kaplunovsky and J. Louis, Nucl. Phys. B355 (1991) 649.

[9] J.-P. Derendinger, S. Ferrara, C. Kounnas and F. Zwirner, Nucl. Phys. B372 (1992) 145 and Phys. Lett. 271B (1991) 307;

S. Ferrara, C. Kounnas, D. Lüst and F. Zwirner, Nucl. Phys. B365 (1991) 431;

I. Antoniadis, K.S. Narain and T. Taylor, Phys. Lett. 267B (1991) 37;

G. Lopes Cardoso and B.A. Ovrut, Nucl. Phys. B369 (1992) 351;

I. Antoniadis, E. Gava, K.S. Narain and T. Taylor, Nucl. Phys. B407 (1993) 706 and ibid. B413 (1994) 162;

P. Mayr and S. Stieberger, Nucl. Phys. B407 (1993) 725;

D. Bailin, A. Love, W.A. Sabra and S. Thomas, Mod. Phys. Lett. A9 (1994) 67;

V. Kaplunovsky and J. Louis, Nucl. Phys. B444 (1995) 191.

[10] E. Kiritsis and C. Kounnas, Nucl. Phys. B41 [Proc. Sup.] (1995) 331; Nucl. Phys. B442 (1995) 472; proceedings of STRINGS 95, Future Perspectives in String Theory, Los Angeles, CA, 13-18 March 1995, hep-th/9507051.

[11] D. Bailin, A. Love, W.A. Sabra and S. Thomas, Mod. Phys. Lett. A10 (1995) 1337.

[12] J.A. Harvey and G. Moore, Nucl. Phys. B463 (1996) 315. 
[13] P.M. Petropoulos and J. Rizos, Phys. Lett. 374B (1996) 49.

[14] E. Kiritsis, C. Kounnas, P.M. Petropoulos and J. Rizos, hep-th/9605011.

[15] E. Kiritsis, C. Kounnas, P.M. Petropoulos and J. Rizos, hep-th/9608034.

[16] I. Antoniadis, E. Gava and K.S. Narain, Phys. Lett. 283B (1992) 209 and Nucl. Phys. B383 (1992) 93.

[17] J.H. Schwarz, Phys. Lett. 371B (1996) 223.

[18] G. Aldazabal, A. Font, L.E. Ibánẽz and F. Quevedo, hep-th/9602097. 